\title{
Disulfiram Implantation for the Treatment of Alcoholism: Clinical Experiences from the Plastic Surgeon's Point of View
}

\author{
Billur Sezgin ${ }^{1}$, Serhat Sibar ${ }^{2}$, Hakan Bulam ${ }^{3}$, Kemal Findikcioglu², Serhan Tuncer ${ }^{2}$, Bilge Dogan ${ }^{4}$ \\ ${ }^{1}$ Department of Plastic, Reconstructive and Aesthetic Surgery, Erzurum Regional Training and Research Hospital, Erzurum; ${ }^{2}$ Department of \\ Plastic, Reconstructive and Aesthetic Surgery, Gazi University Hospital, Ankara; ${ }^{3}$ Department of Plastic, Reconstructive and Aesthetic \\ Surgery, Numune Research and Training Hospital, Ankara; ${ }^{4}$ Department of Psychiatry, Gazi University Hospital, Ankara, Turkey
}

Background Disulfiram implantation is a widely used treatment alternative for alcohol abuse, yet reports on the surgical aspect of disulfiram implantation with respect to patient and drug-related treatment efficacy and wound complications are very limited. We present our clinical experiences with disulfiram implantation and discuss the surgical outcomes obtained with different anatomical planes for implantation.

Methods Medical records of all patients referred to our clinic from the psychiatry department between 2007 and 2013 for disulfiram implantation were retrospectively analyzed. Implantation was carried out using 10 sterile Disulfiram tablets (WZF Polfa S.A.), each tablet containing $100 \mathrm{mg}$ of disulfiram. The procedure was carried out by implanting the tablets randomly in either a subcutaneous or an intramuscular plane. The location and the plane of implantation and the complications were recorded for each patient and compared to determine the differences in the outcomes.

Results A total of 32 implantation procedures were evaluated for this study. Twenty-five implants were placed in the intramuscular plane (78.2\%), while seven implants were placed subcutaneously (21.8\%). Exposure was encountered in three of the seven subcutaneous implants (42.9\%), while no exposure was seen with the intramuscular implants. Incomplete absorption of the tablets was encountered in one patient with a previous subcutaneous implant who presented 1 year later for re-implantation as part of the continuation of therapy. Conclusions To overcome the issue of treatment continuation in the case of disulfiram therapy, which may be ceased due to frequently encountered wound complications, we believe that implantation in the subscapular intramuscular plane allows both uneventful healing and an out-of-reach implant location.

Keywords Alcoholism / Bioabsorbable implant / Disulfiram
Correspondence: Billur Sezgin Department of Plastic, Reconstructive and Aesthetic Surgery, Erzurum Regional Training and Research Hospital, Cat yolu 25070 Erzurum, Turkey

Tel: +90-312-266-1167

E-mail: billursezgin@hotmail.com

This study was presented at the 35 th Congress of the Turkish Society of Plastic, Reconstructive and Aesthetic Surgeons on October 28-31, 2013, in Istanbul, Turkey.

No potential conflict of interest relevant to this article was reported.

Received: 27 May 2014 • Revised: 3 Jul 2014 • Accepted: 12 Jul 2014

pISSN: 2234-6163 • elSSN: 2234-6171 • http://dx.doi.org/10.5999/aps.2014.41.5.571 • Arch Plast Surg 2014;41:571-575

\section{INTRODUCTION}

Due to the widespread devastating consequences of alcohol abuse, a range of pharmacological and non-pharmaceutical interventions has been developed to control alcohol-related problems. One of the oldest pharmacological agents used for this

Copyright $(\subseteq 2014$ The Korean Society of Plastic and Reconstructive Surgeons

This is an Open Access article distributed under the terms of the Creative Commons Attribution Non-Commercial License (http://creativecommons.org/

licenses/by-nc/3.0/) which permits unrestricted non-commercial use, distribution, and reproduction in any medium, provided the original work is properly cited.

www.e-aps.org 
purpose is disulfiram.

Tetraethylthiuram disulfide (disulfiram) dates back to the 1800 s, when it was first used in the manufacturing process of rubber. Its potential deterrent effect from alcohol consumption was discovered in 1937, when a chemical plant physician named Williams [1] reported the unpleasant symptoms encountered by disulfiram-exposed workers after alcohol consumption. Ten years later, Hald and Jacobsen [2] observed similar reactions while working with disulfiram as a vermicide. They collaborated with Martensen-Larsen [3] in clinical trials which proved disulfiram to be a promising agent for the pharmacological treatment of alcohol abuse.

Disulfiram acts by producing an acute reaction following alcohol intake. It blocks the enzyme acetaldehyde dehydrogenase, which results in a metabolic build-up of acetaldehyde, thus creating a series of unpleasant symptoms after alcohol consumption referred to as the disulfiram-ethanol reaction (DER) [4]. The physical discomfort is quite unbearable; therefore, upon experiencing these symptoms, most people immediately cease alcohol intake. Although it is a widely used treatment alternative, the main concern regarding disulfiram therapy is the difficulty of getting patients to take disulfiram on a regular basis. Therefore, techniques to increase treatment adherence are practiced, including supervision and disulfiram implantation [5].

Subcutaneous implantation of disulfiram was first described in 1968 [6]. The effectiveness of disulfiram implants with respect to their pharmacological effects remains questionable due to the lack of blood level monitoring, yet studies have shown that they produce a significant increase in abstinence, most likely due to psychological deterrence $[7,8]$. However, reports on the surgical aspect of disulfiram implants with respect to patient and

\section{Fig. 1. Site of implantation}

The subscapular area was preferred for disulfiram implantation as it is out-of-sight and out-of-reach for patients.

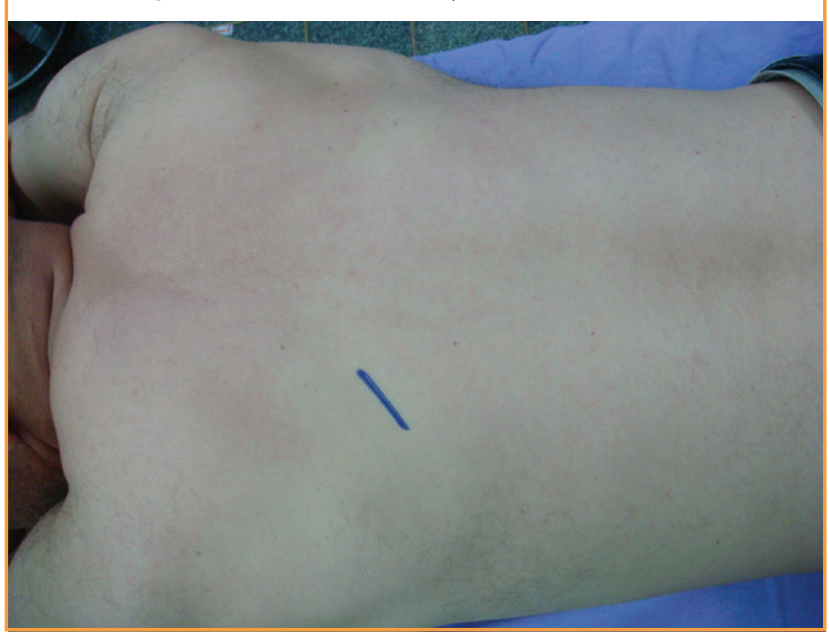

drug-related treatment efficacy and wound complications are very limited $[9,10]$.

We present our clinical experiences with disulfiram implantation and discuss the surgical outcomes obtained with different anatomical planes for implantation.

\section{METHODS}

Medical records of all patients referred to our clinic from the psychiatry department between 2007 and 2013 for disulfiram implantation were retrospectively analyzed for this study. Implantation was carried out using 10 sterile Disulfiram tablets (WZF Polfa S.A., Warsaw, Poland), each tablet containing 100 mg of disulfiram. The procedure was carried out by implanting the tablets randomly in either a subcutaneous or an intramuscular plane. Under local anesthesia and sterile conditions, a $3-\mathrm{cm}$ incision was made subscapularly, and following dissection, a subcutaneous or intramuscular pouch of approximately $3 \mathrm{~cm} \times 3$ $\mathrm{cm}$ was created in the dorsal region of each patient (Fig. 1). The latissimus dorsi muscle was split parallel to its fibers in order to create a pouch in the cases where an intramuscular plane was desired (Fig. 2). Following hemostasis, all tablets were placed in the pouch and closure was achieved with layered suturing (Fig. 3). Patients were followed up by the plastic surgery clinic for suture removal two weeks later and for a one-month wound check.

The dorsal subscapular area was chosen for all implants as it was the area least likely to be seen or reached by the patient on a daily basis. The location and the plane of implantation and the complications were recorded for each patient and compared to determine the differences in the outcomes.

Fig. 2. Intramuscular disulfiram implantation technique

For intramuscular implants, the latissimus dorsi muscle was split parallel to its fibers and dissected to create a $3 \mathrm{~cm} \times 3 \mathrm{~cm}$ intramuscular pouch.

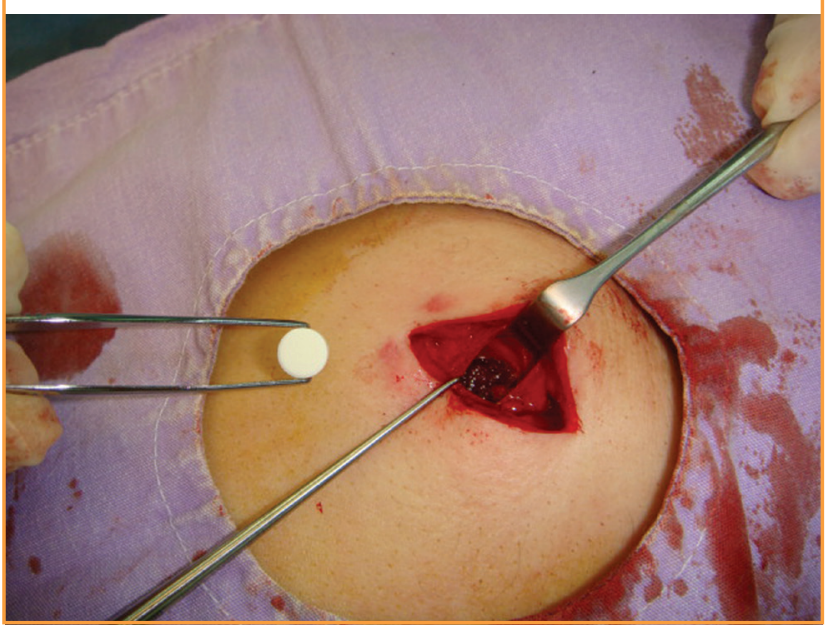




\section{Fig. 3. Implanted disulfiram tablets}

Ten sterile disulfiram tablets were implanted inside the pouch.

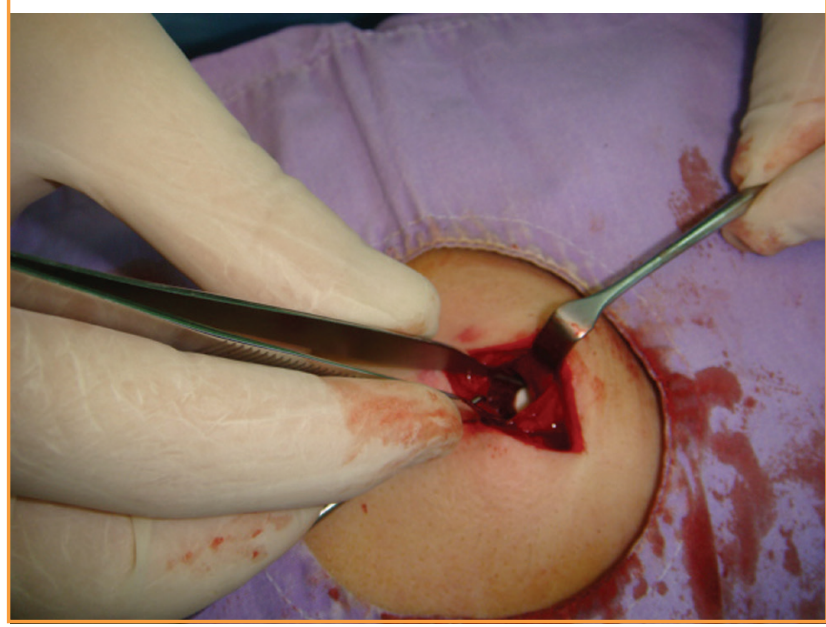

Table 1. Distribution of disulfiram implants reviewed in the study

\begin{tabular}{lccc|}
\hline $\begin{array}{l}\text { Type of } \\
\text { implantation }\end{array}$ & Subcutaneous & Intramuscular & Total \\
\hline Single implantation & 7 & 17 & 24 \\
Second implantation ${ }^{\text {a) }}$ & 0 & 8 & 8 \\
Total & 7 & 25 & 32 \\
\hline
\end{tabular}

a) Of the eight patients receiving a second implant, two were due to tablet exposure following subcutaneous implantation while six were for the continuation of treatment.

\section{RESULTS}

A total of 24 male patients were included in the study. The mean age of the patients was 40.5 years (range, 27-55 years). Eight (33.3\%) patients had undergone the implantation procedure twice, while 16 (67.7\%) patients had a record of single implantation. Of the patients undergoing two procedures, six had the procedure repeated one year after for the continuation of treatment, while two patients suffered from implant exposure and were re-implanted immediately after wound healing. Therefore, a total of 32 implantation procedures were evaluated (Table 1).

Twenty-five implants were placed in the intramuscular plane (78.2\%), while seven implants were placed subcutaneously (21.8\%). All subcutaneous implantations were carried out only in the first two years of the study, while the intramuscular plane was preferred throughout the study period. This was due to the high complication rates encountered with subcutaneous implants within the first two years of disulfiram implantation, which led us to discontinue the use of this plane and opt for the intramuscular plane exclusively. As the subcutaneous group had very few patients to conduct an analysis, a statistical comparative study could not be carried out.

\section{Fig. 4. A case of incomplete disulfiram absorption}

Incomplete absorption of tablets was noted in a patient with previous subcutaneous implants while being implanted for a second course of disulfiram treatment.

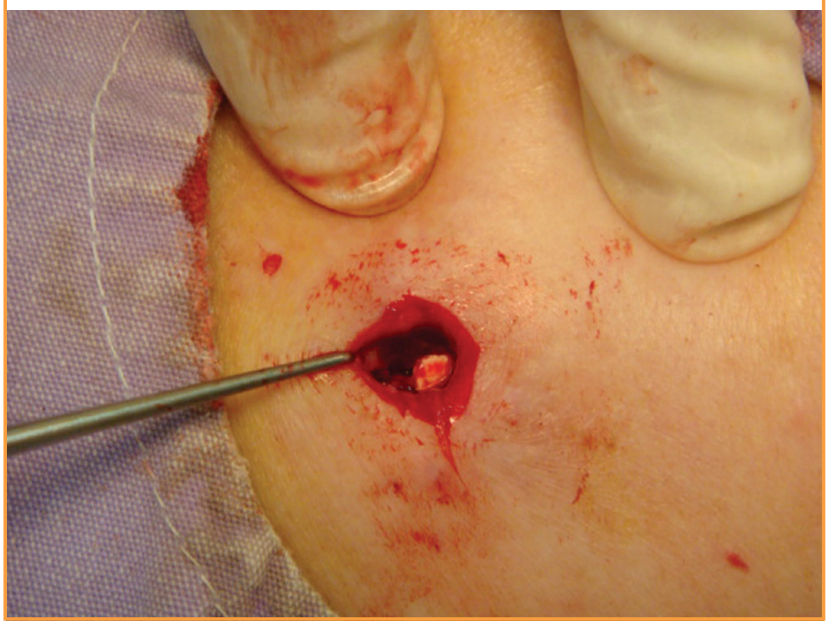

\section{Fig. 5. Incomplete disulfiram absorption}

Four out of the 10 tablets were not fully absorbed during the oneyear treatment of subcutaneous disulfiram implantation.

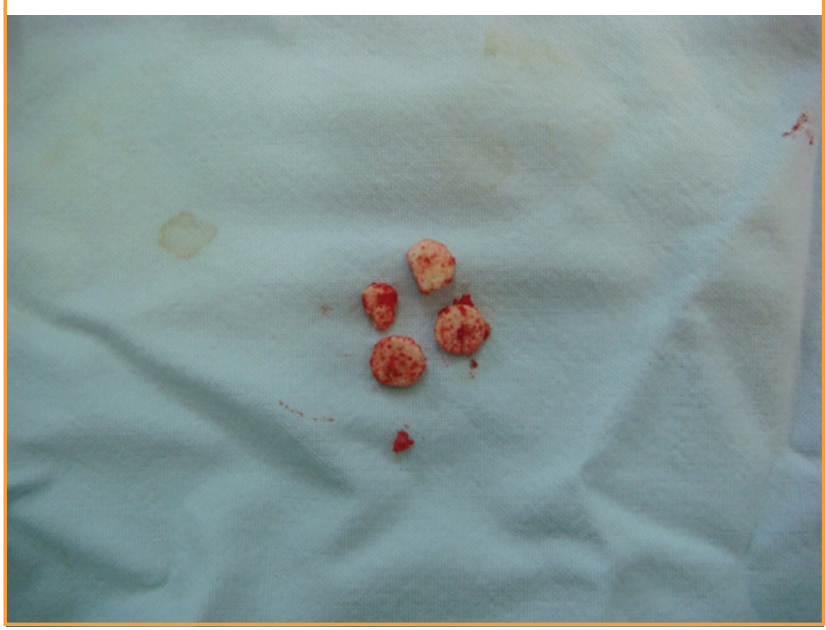

Exposure was encountered in three of the seven subcutaneous implants (42.9\%), while no exposure was seen in the case of the intramuscular implants. Implant extrusion resulted in the cessation of the disulfiram treatment for one patient. The two other patients who presented with exposure and wished to continue treatment were re-implanted in the intramuscular plane following the healing of the previous wound. Patients with intramuscular implantation complained of a short duration of mild pain, which resolved completely within a few weeks.

Patients that presented one year later for re-implantation for the continuation of therapy were operated on through the same incision. Incomplete absorption of the tablets was encountered in one patient with a previous subcutaneous implant (Figs. 4, 5). This patient was also re-implanted with intramuscular tablets. 
Complete resorption of the tablets was noted in the other seven patients.

Since blood level monitoring is not applicable for disulfiram, patients were routinely followed-up by the psychiatry department to determine the duration of abstinence. None of the patients experienced a DER as abstinence was attained by all the patients included in the study during the disulfiram treatment.

\section{DISCUSSION}

Disulfiram treatment is a widely accepted treatment alternative in alcohol use disorders both for its pharmacological effects through the DER and as a psychological deterrent. Literature studies state that although the pharmaceutical efficacy of implantation is not standard due to unsupervised blood levels, there is a significant increase in the duration of abstinence when compared with the placebo [7]. Therefore, disulfiram implants remain a highly chosen treatment alternative for alcohol abuse.

Although disulfiram implantation was first described as a subcutaneous procedure and many studies report subcutaneous implantation $[6,8,9]$, there have been limited studies addressing wound complications for disulfiram implants and the effect that these complications may have on the overall treatment. Johnsen et al. [9] reported exposure in 3 out of 10 patients following subcutaneous implantation in the abdominal region. Malcolm and Madden [10] reported wound breakdown and extrusion of tablets in 13 of the 70 abdominal intramuscular implantations in their study. Although both studies reported wound breakdown and implant exposure as the most common and troublesome complication, neither of the studies evaluated or addressed the surgical aspect or implantation plane as a solution to this problem.

Our study demonstrated a higher level of complications with subcutaneous implantations where one patient discontinued disulfiram treatment and two other patients had to undergo a time period without disulfiram therapy for the wound to resolve in order to receive another implantation procedure. After encountering exposure with subcutaneous implants, we started using the intramuscular plane exclusively and since then, have not had cases of implant exposure.

There are other treatment modalities that are administered by subcutaneous implantation. Hormonal therapies constitute a wide portion of these treatments such as progestin-containing rods and testosterone in a subcutaneous pellet form $[11,12]$. The implantation of these medications differs from that of disulfiram as they are introduced into the subdermal arm or thigh region with the help of trocars. A similar approach could be adopted for disulfiram implants, yet the main difference between hormonal therapies and disulfiram therapy is the difficulty that can be faced with patients trying to overcome an addiction disorder. Extreme measures may be taken by the patients if they have the urge to consume alcohol; therefore, we believe that the placement of implants should be out-of-reach and out-of-sight for this patient population. Although there are records of gluteal, abdominal, and dorsal implantations in the literature, we implant all patients intramuscularly in the subscapular region for this reason. The subscapular area is also anatomically confined in comparison to other areas, which we believe contributes to the protection of the area from external trauma and minimizes the awareness of carrying an implant on a daily basis, which could have a negative impact on the patient's psychological well-being. Subcutaneous implantation with trocars may reduce the risk of infection and could also prove to be an easier and more effective approach, provided it is undertaken in an out-of-reach location.

In conclusion, in order to overcome the main issue of disulfiram treatment, which is adherence to therapy, which may be ceased in the case of disulfiram implantation due to frequently encountered wound complications, we believe that implantation in the subscapular intramuscular plane allows both uneventful healing and an out-of-reach implant location. This is the first detailed report on the effect of the implantation plane of disulfiram on wound complications and treatment efficacy.

\section{REFERENCES}

1. Williams EE. Effects of alcohol on workers with carbon disulfide. JAMA 1937;109:1472-3.

2. Hald J, Jacobsen E. A drug sensitizing the organism to ethyl alcohol. Lancet 1948;2:1001-4.

3. Martensen-Larsen O. Treatment of alcoholism with a sensitizing drug. Lancet 1948;2:1004.

4. Johansson B. A review of the pharmacokinetics and pharmacodynamics of disulfiram and its metabolites. Acta Psychiatr Scand Suppl 1992;369:15-26.

5. Allen JP, Litten RZ. Techniques to enhance compliance with disulfiram. Alcohol Clin Exp Res 1992;16:1035-41.

6. Kellam AM, Wesolkowski JM. Disulfiram implantation for alcoholism. Lancet 1968;1:925-6.

7. Wilson A, Davidson WJ, White J. Disulfiram implantation: placebo, psychological deterrent, and pharmacological deterrent effects. Br J Psychiatry 1976; 129:277-80.

8. Johnsen J, Morland J. Disulfiram implant: a double-blind placebo controlled follow-up on treatment outcome. Alcohol Clin Exp Res 1991;15:532-6.

9. Johnsen J, Stowell A, Bache-Wiig JE, et al. A double-blind placebo controlled study of male alcoholics given a subcutaneous disulfiram implantation. Br J Addict 1987;82:607-13. 
10. Malcolm MT, Madden JS. The use of disulfiram implantation in alcoholism. Br J Psychiatry 1973;123:41-5.

11. Beasley A, Schutt-Aine A. Contraceptive procedures. Obstet Gynecol Clin North Am 2013;40:697-729.
12. Cavender RK, Fairall M. Subcutaneous testosterone pellet implant (Testopel) therapy for men with testosterone deficiency syndrome: a single-site retrospective safety analysis. J Sex Med 2009;6:3177-92. 